\title{
REDIRECTION OF PARA-SAGITTAL CUTS OF MAGNETIC RESONANCE IMAGING FOR TEMPOROMANDIBULAR JOINT
}

\author{
Mohamed Khalifa Zayet *
}

\begin{abstract}
Background: Magnetic resonance imaging (MRI) is the main imaging modality in the diagnosis of temporomandibular disorders. Furthermore, sagittal cuts are the chief plane in diagnosis of these disorders.
\end{abstract}

Aim of the study: This study aimed to investigate the validity of para-sagittal cuts taken parallel to lateral pterygoid muscle in depicting the structures of temporomandibular joint.

Subjects and Methods: Twenty symptomatic temporomandibular joint (TMJ) patients performed MRI bilaterally using the traditional para-sagittal cuts and another plane parallel to lateral pterygoid muscle. Four-point scale was used to assess the clarity of articular disc position, disc morphology, cortical bone, and overall quality of the images of both investigated techniques in closed and open positions. Moreover, the final diagnosis of the cases was obtained in each technique.

Results: The mean and standard deviation of muscular aligned cuts in clarity of revealing disc position, disc position, cortical bone, and overall image quality in closed position were 2.83 $\pm 0.39,2.63 \pm 0.49,2.53 \pm 0.51$, and $2.83 \pm 0.39$, respectively, while those for traditional plane were $2.55 \pm 0.50,2.43 \pm 0.50,2.80 \pm 0.41$, and $2.53 \pm 0.51$ with the same order. In open position, the same investigated points got $2.80 \pm 0.41,2.73 \pm 0.45,2.73 \pm 0.45$, and $2.80 \pm 0.41$ in muscular aligned images and $2.78 \pm 0.42,2.65 \pm 0.48,2.82 \pm 0.39$, and $2.78 \pm 0.42$ in the traditional plane. Both techniques provided the same diagnosis in $97.5 \%$ of the cases.

Conclusion: Parasagittal magnetic resonance images taken parallel to lateral pterygoid muscle deposited higher image quality than the traditional plane in revealing temporomandibular structures.

KEYWORDS: TMJ - MRI - lateral pterygoid muscle

\section{INTRODUCTION}

Temporomandibular disorders (TMD) are among the common problems in dental practice. They constitute $20-40 \%$ of the causes of oro-facial pain, which is considered a large percentage when compared with dental problems that constitute $50 \%$ of them ${ }^{(1,2)}$. Temporomandibular disorders as a whole are one of the diagnostic dilemmas that face the dentists because of its diversity ${ }^{(3)}$. Despite that history taking and clinical examination may be

* Ass. Professor, Oral and Maxillofacial Radiology, Faculty of Dentistry, Cairo University 
beneficial in a lot of cases, but sometimes this is not enough due to overlap of signs and symptoms that occurs in many cases, especially long standing ones. Hence, the need for one of the radiographic examination modalities to complete the profile of the disease and subsequently aids in the diagnosis of the case was mandatory ${ }^{(4)}$. Magnetic Resonance Imaging (MRI) due to its superb contrast resolution of soft tissue is considered the gold standard imaging tool in dealing with temporomandibular disorders ${ }^{(5)}$.

Magnetic resonance imaging as a cross-sectional imaging modality has the ability to provide images in axial, sagittal, and coronal planes ${ }^{(6)}$. Nevertheless, sagittal cuts are the main element in the three orthogonal planes to diagnose temporomandibular disorders. The inclination of the cuts is the most crucial point in revealing the relationship between the articulating surfaces properly. The traditional direction that used for decades is to take these cuts perpendicular the long axis of the condyle ${ }^{(7)}$. This traditionally used plane has a historical background, where the posterior inclination of each condyle was an influencing factor in imaging of TMJ. This influence started with plain radiographic techniques, like transcranial and trans-orbital techniques (8,9) and flourished with conventional tomography ${ }^{(10)}$, and extended to contemporary X-ray based crosssectional techniques like multislice and cone beam computed tomography ${ }^{(11,12)}$ and even non-ionizing radiation dependent modalities, like MRI (13). In some cases the outline of the condyle is not clear enough in axial MR images ${ }^{(14)}$, this lack of clarity is due to either changes in the condyle itself or due to insufficient quality of localizing axial cuts.

Lateral pterygoid muscle is the most relevant one to the temporomandibular joint as the inferior belly is inserted to the neck of the condyle, while some fibers of the superior belly are attached to the articular disc ${ }^{(15,16)}$. On the other side, it originates from the lateral surface of lateral pterygoid plate and infra-temporal surface of greater wing of sphenoid bone ${ }^{(17)}$. The size and the orientation of the lateral pterygoid muscle render it easier to be identified on axial MR images ${ }^{(18)}$.

The aim of this study was to investigate the accuracy of para-sagittal MR images that are taken parallel to the lateral pterygoid muscle in revealing temporomandibular structures.

\section{SUBJECTS AND METHODS}

This prospective diagnostic accuracy study was conducted on 40 temporomandibular joints in 20 patients. They were 19 females and one male. Their age ranged from 17 to 39 years with average 26.8 years. The inclusion criteria involved patients with TMJ related signs and symptoms and indicated for MRI scanning; such as TMJ sounds, pain or tenderness at the joint region, or limited mouth opening. The exclusion criteria included patients with TMJ related surgeries or patients with previous maxillofacial traumatic injuries, or those who cannot perform MRI scan based on pre-operative MRI examination questionnaire ${ }^{(19)}$ like those with pacemaker, cerebral aneurysm clips, intra-ocular or periocular foreign body, claustrophobic patients, and pregnant females. This study was approved by Research ethics Committee of Faculty of Dentistry, Cairo University. Besides, written informed consent was taken from each patient, the consent involved the diagnostic benefits of the research and possible complications, as well as confidentiality of the data. The procedures of MRI scan with necessary instructions were explained for the patients.

MRI scanning: patients were scanned at Radiodiagnosis outpatient clinic in Kasr El-Einy hospital using 1.5 Tesla superconductive magnet (Philips, Intera, Netherlands). The investigator attended all MRI procedures to check the quality of the scans and to inform the patients what to do during the MRI scanning.

MRI scan preparation: removable metallic objects like removable partial dentures or vitallium, 
earrings, necklace or sweater with zipper were removed before the beginning of the scan. Scout MRI scan was performed at first, followed by axial $\mathrm{T} 2$ turbo spin echo sequence, on which planning of para-sagittal cuts was performed. Proton densityturbo spin echo para-sagittal cuts were taken twice; one with the traditional direction; perpendicular to the transverse long axis of each condyle (perpendicular to the line between medial and lateral poles of the condyle) and the other direction was parallel to the fibers of lateral pterygoid muscle in the anteroposterior direction. Both para-sagittal cuts were done while the patient occluding in centric occlusion then in maximum opening using tongue depressors (Fig. 1). The number of tongue depressors was determined on individual basis before the scanning. The four sequences were taken with standardized parameters which were as following: repetition time (TR): $1500 \mathrm{msec}$, echo time (TE): $30 \mathrm{msec}$, slice thickness: $2.5 \mathrm{~mm}$, inter slice gap: $0.3 \mathrm{~mm}$, FOV: $150 \mathrm{~mm}$, frequency matrix: 256 , phase matrix: 256 , NEX: 3 Number of slices: 11, stacks:2 scanning time 2:50 min. with application of TMJ surface coil.

Image analysis: Images were printed on (11 x 14 inch) LASER films using LASER printer (DryPix 4000, Fujifilm - USA). Images were assessed on a viewing box by an oral and maxillofacial radiologist with 17-year experience. The assessment was done blindly after covering the scanning data on the films by an opaque sheet, and with haphazard order of the films. Every assessment session was 45 minutes maximum and would be ended in case of occurrence of eye or mental fatigue. A four-point scale was used to evaluate the quality of the images regarding clarity and visibility of the following points: articular disc position, disc morphology, cortical bone, and overall image quality with the following order: $0=$ Poor images, $1=$ Fair images, $2=$ Good images, $3=$ Very good images. Moreover, the final diagnosis of each case, whether it is normal, anterior disc displacement with reduction or anterior disc displacement without reduction, was determined through images of closed and open positions.

Statistical analysis: Data were statistically described in terms of mean \pm standard deviation ( \pm SD). Comparison between the traditional and muscular aligned techniques as well as between closed and open positions was done using Wilcoxon signed rank test for paired (matched) samples. Comparison of final diagnosis was done using McNemar test and agreement was tested using weighted kappa statistic. Two sided $p$ values less than 0.05 was considered statistically significant. All statistical calculations were done using computer program IBM SPSS (Statistical Package for the Social Science; IBM Corp, Armonk, NY, USA) release 22 for Microsoft Windows.
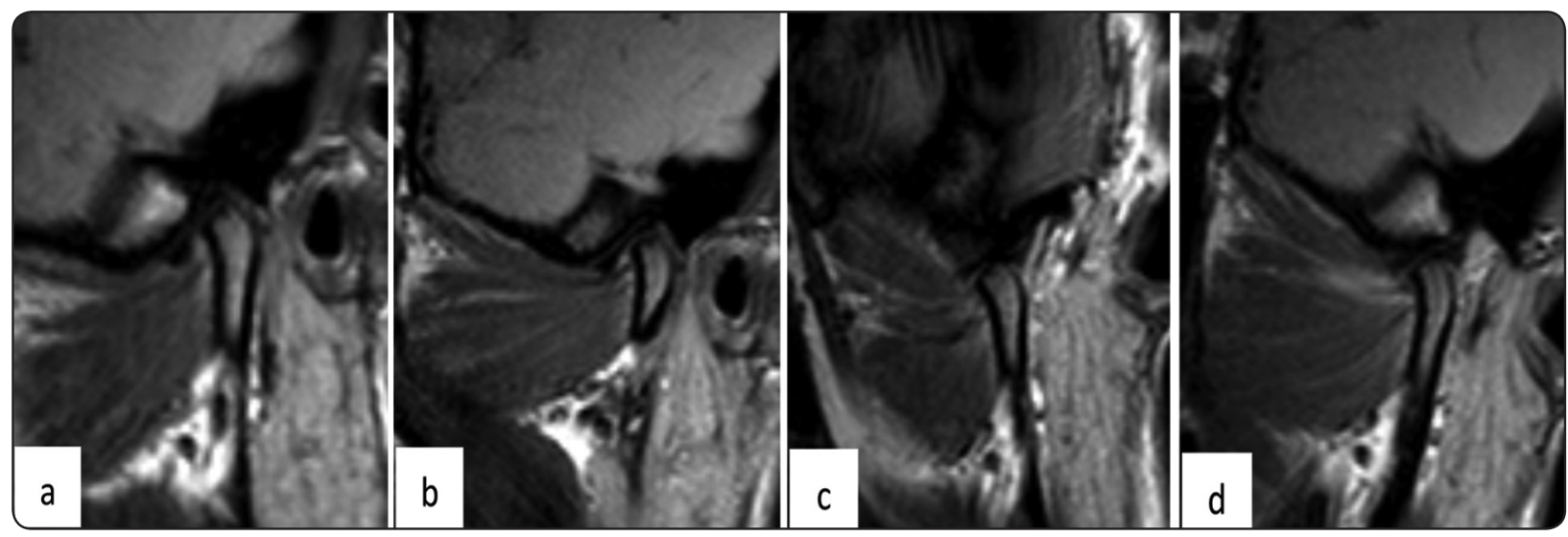

Fig. (1): Para-sagittal proton density turbo spin echo MR images in (a): traditional plane (closed), (b): muscular aligned plane (close), (c): traditional plane (open), and (d): muscular aligned plane (open). 


\section{RESULTS}

This study was conducted by on 40 temporomandibular joints in 20 patients by taking para-sagittal cuts in two directions: traditional and muscular aligned in both closed and open positions for clarity of disc position, disc morphology, cortical bony surfaces, and overall image quality.

Regarding closed position the mean and standard deviation of the traditional direction in the clarity of revealing articular disc position was $2.55 \pm 0.50$, while in muscular aligned direction it was $2.83 \pm$ 0.39 . With respect to disc morphology, the mean and standard deviation of traditional technique was $2.43 \pm 0.50$ and $2.63 \pm 0.49$ in muscular aligned technique. Both differences were statistically significant with $p$ value: 0.012 for disc position and 0.033 for disc morphology.

The clarity of cortical bone was statistically higher in traditional technique ( $p$ value: 0.008 ), where the mean of scores and standard deviation were $2.80 \pm 0.41$ in traditional technique and $2.53 \pm$ 0.51 in the new technique. The overall image quality of muscular aligned technique was significantly higher than the traditional technique ( $p$ value: $0.005)$, with mean and standard deviation 2.83 \pm 0.39 and 2.53 \pm 0.51, respectively (Fig. 2).

Regarding open position, the clarity of disc position and disc morphology had the scores of $2.78 \pm 0.42$ and $2.65 \pm 0.48$ respectively in traditional

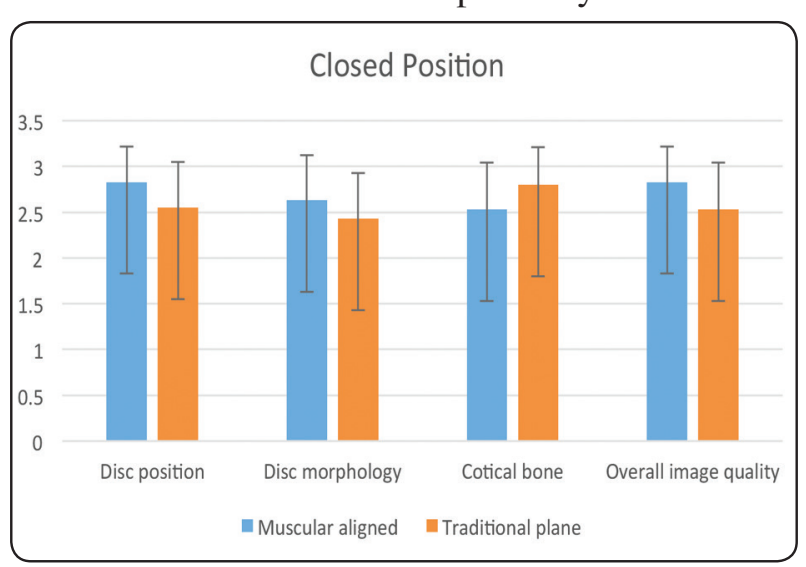

Fig. (2): Bar-chart showing mean and standard deviation of muscular and traditional plane in closed position. direction. While in muscular aligned direction, they were $2.80 \pm 0.41$ and $2.73 \pm 0.45$ with the same order. The difference was non-significant statistically in both of them ( $\mathrm{p}$ value: 0.655 for disc position and 0.257 for disc morphology). Although traditional technique got higher results in delineation of cortical bone $(2.82 \pm 0.39)$ when compared to muscular aligned technique $(2.73 \pm 0.45)$, but this difference was non-significant statistically ( $p$ value: 0.366 ). For overall image quality there was no statistical significant difference between both techniques ( $p$ value: 0.655$)$, where the traditional one got mean and standard deviation $(2.78 \pm 0.42)$ and the new

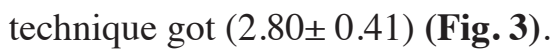

Regarding comparison between closed and open positions in the same technique, statistical analysis revealed significant difference in the favor of open position in depicting disc position, disc morphology, and overall quality of traditional technique with $p$ values: $0.013,0.020$, and 0.008 , respectively. While for muscular aligned technique the significant difference was in the favor of open position in revealing cortical bone ( $p$ value: 0.011 )

With regard to the final diagnosis obtained through closed and open positions of both techniques, they provided the same diagnosis in 97.5\% of cases, McNemar Bowker test revealed non-significant difference and weighted kappa test showed correlation coefficient of agreement of 0.962 .

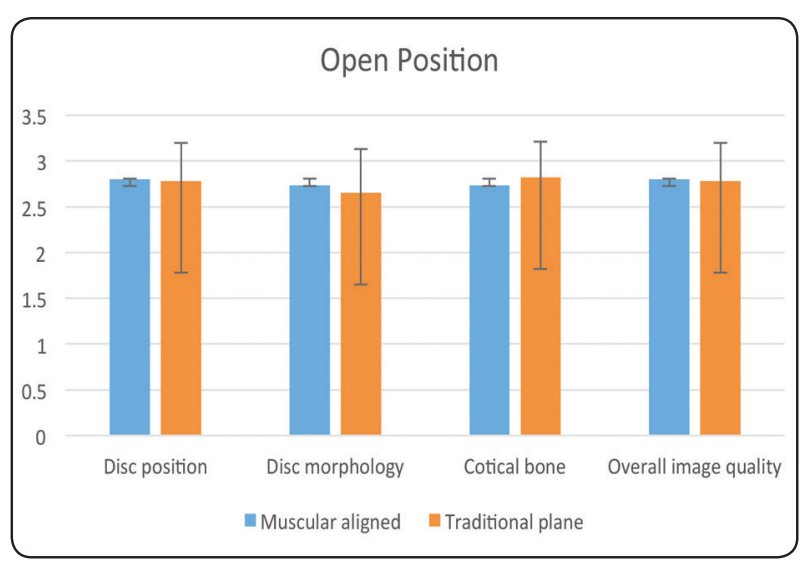

Fig. (3): Bar-chart showing mean and standard deviation of muscular and traditional plane in open position. 


\section{DISCUSSION}

In MRI imaging of temporomandibular joint, the para-sagittal cuts taken parallel to the direction of lateral pterygoid muscle antero-posteriorly had comparable and sometimes better results than those taken perpendicular to horizontal long axis of the condyles in revealing TMJ structures of diagnostic value.

The objective of the current study was based on the hypothesis whether there is another plane that could be used in diagnosis of temporomandibular disorders through magnetic resonance images. The need for different para-sagittal plane in magnetic resonance imaging of TMJ was based on few points. Firstly, MRI is the lonely imaging modality capable of revealing articular disc directly and with sufficient degree of clarity. Therefore, the articular disc should be the focus of slicing instead of the condyle. Secondly, the outline of condyles with arthritic changes or considerable deformity may not be obvious enough in localizer cuts, especially in long standing cases and accordingly, determination of its horizontal long axis may be difficult or even not accurate. Thirdly, sometimes, in order to reduce the scanning time, the parameters of the localizer axial view are not high enough. Consequently, the image quality of these cuts is not adequately high for proper and accurate planning of para-sagittal cuts.

Delineation of articular disc is of paramount importance in interpretation of magnetic resonance images for TMJ, as its position in the closed position is considered a crucial differentiating point between intra-capsular and muscular disorders ${ }^{(20)}$, and its position in open position determines the type of internal derangement ${ }^{(21)}$. Moreover, its form has a considerable share in the prognosis of the case. Statistical analysis revealed that the clarity of disc position as well as disc morphology are higher in muscular aligned cuts than traditional sagittal cuts with significant difference. This difference could be attributed to the point that muscular aligned cuts are more medially inclined than condylar dependent cuts. The reason behind clarity of disc in that plane is the frequent presence of medial component of disc displacement beside anterior one ${ }^{(22)}$, where anatomically the space between mandibular and temporal bony components of TMJ is wider medially ${ }^{(23)}$. The second reason is in normal joint, the disc is stabilized circumferentially by ligaments, retro-discal tissues, and fibers of superior belly of lateral pterygoid muscle ${ }^{(24)}$. However, in diseased joint the ligaments become elongated and loose, retro-discal tissues may lose its capability to retract the disc. Therefore, lateral pterygoid muscle may have the upper hand in directing the disc with its medial pull.

In open position, the difference between both techniques was not significant statistically in depiction of disc related outcomes (disc position and disc morphology). This finding may be due to the shifting of the condyle medially during opening with the action of lateral pterygoid muscle, especially with unilateral joint affection, or presence in difference of reducibility of the disc in both sides, which was found in multiple cases in the current study.

Regarding delineation of cortical bone in articulating part of the joint, it was expected that traditional plane is superior to the new technique, as the former is planned according to exact inclination of each condyle, which was found in this study. However, this superiority was statistically significant in closed position only. On the other hand, the relation between the condyle and the articular disc in normal and reducible cases rendered the difference in delineation non-significant in open position.

The previous results pour in the favor of muscular aligned images especially in closed 
position, which was confirmed statistically, where the overall image quality was significantly higher in the new investigated technique, but both techniques provided nearly the same results in the open position. Moreover, the new technique provided the same diagnosis of the old one in 39 cases out of 40 $(97.5 \%)$, which denotes that muscular aligned cuts could be an accurate alternative to conventionally used plane in diagnosis of TMD through magnetic resonance images.

The main limitation of the current study lies in the heterogeneity of the sample between normal and abnormal joints, reducible and non-reducible discs, and unilateral and bilateral affection. Therefore, the results may differ if the following studies have more homogenous criteria and are conducted on larger sample size. However, within the limitations of the present study, it is recommended to use the muscular aligned para-sagittal cuts, especially in cases with malformed condyles, or scans with multiple requested pulse sequences, or MRI scanners with low magnetic strength in the scanning of patients with temporomandibular disorders.

\section{CONCLUSION}

In magnetic resonance imaging of temporomandibular joint, para-sagittal cuts taken parallel to the antero-posterior direction of lateral pterygoid muscle provide higher image quality and better depiction of articular disc position and morphology than traditionally applied para-sagittal plane, especially in closed position.

\section{Conflict of interest:}

The author reports no conflict of interest

\section{ACKNOWLEDGEMENT}

Great gratitude to Mrs. Hala Ali, MRI technician in Kasr El-Einy Hospital, for her efforts in scanning procedures in the current study.

\section{REFERENCES}

1. Fernandes G, Gonçalves DAG, Conti P. Musculoskeletal Disorders. Dent Clin North Am. 2018 Oct;62(4):553-564. Review.

2. Racich MJ. Occlusion, temporomandibular disorders, and orofacial pain: An evidence-based overview and update with recommendations. J Prosthet Dent. 2018 Nov;120(5):678-685. Review.

3. Okeson JP, de Leeuw R. Differential diagnosis of temporomandibular disorders and other orofacial pain disorders. Dent Clin North Am. 2011 Jan;55(1):105-20. Review.

4. Klasser GD, Okeson JP. Role of the dentist in the management of orofacial pain. Pain Manag. 2015;5(6): 407-11.

5. Talmaceanu D, Lenghel LM, Bolog N, Hedesiu M, Buduru S, Rotar H, Baciut M, Baciut G. Imaging modalities for temporomandibular joint disorders: an update. Clujul Med. 2018 Jul;91(3):280-287. Review.

6. White, S. C. and Pharoah, M. J. (2014) Oral radiology : Principles and Interpretation. $7^{\text {th }}$ ed. Elseiver Mosby.

7. Yang ZJ, Song DH, Dong LL, Li B, Tong DD, Li Q, Zhang FH. Magnetic resonance imaging of temporomandibular joint: morphometric study of asymptomatic volunteers. J Craniofac Surg. 2015 Mar;26(2):425-9.

8. Cobo J, Argüelles J, Vijande M, Costales M, Fernández Y. Transcranial oblique lateral radiography to verify the position of the mandibular condyles with the use of functional appliances. Eur J Orthod. 1993 Oct;15(5): 387-91.

9. Lewis EL, Dolwick MF, Abramowicz S, Reeder SL. Contemporary imaging of the temporomandibular joint. Dent Clin North Am. 2008 Oct;52(4):875-90. Review.

10. Larheim TA. Comparison between three radiographic techniques for examination of the temporomandibular joints in juvenile rheumatoid arthritis. Acta Radiol Diagn (Stockh). 1981;22(2):195-201

11. Kaur A, Natt AS, Mehra SK, Maheshwari K, Singh G, Kaur A. Improved Visualization and Assessment of Condylar Position in the Glenoid Fossa for Different Occlusions: A CBCT Study. J Contemp Dent Pract. 2016 Aug 1;17(8):679-86.

12. Ganugapanta VR, Ponnada SR, Gaddam KP, Perumalla K, Khan I, Mohammed NA. Computed Tomographic 
Evaluation of Condylar Symmetry and Condyle-Fossa Relationship of the Temporomandibular Joint in Subjects with Normal Occlusion and Malocclusion: A Comparative Study. J Clin Diagn Res. 2017 Feb;11(2):ZC29-ZC33.

13. Vitale GJ, Amato CJ, Falk HJ, Niebloom TA. Magnetic resonance imaging of the temporomandibular joint. J N J Dent Assoc. 1990 Summer;61(3):40-4.

14. Litko M, Szkutnik J, Berger M, Różyło-Kalinowska I. Correlation between the lateral pterygoid muscle attachment type and temporomandibular joint disc position in magnetic resonance imaging. Dentomaxillofac Radiol. 2016 Oct;45(8):20160229.

15. Schmolke C. The relationship between the temporomandibular joint capsule, articular disc and jaw muscles. J Anat. 1994 Apr;184 ( Pt 2):335-45.

16. D'Ippolito SM, Borri Wolosker AM, D'Ippolito G, Herbert de Souza B, Fenyo-Pereira M. Evaluation of the lateral pterygoid muscle using magnetic resonance imaging. Dentomaxillofac Radiol. 2010 Dec;39(8):494-500.

17. Murray GM, Phanachet I, Uchida S, Whittle T. The human lateral pterygoid muscle: a review of some experimental aspects and possible clinical relevance. Aust Dent J. 2004 Mar;49(1):2-8. Review.

18. Ngamsom S, Nakamura S, Sakamoto J, Kotaki S, Tetsumura A, Kurabayashi T. The intravoxel incoherent motion MRI of lateral pterygoid muscle: a quantitative analysis in patients with temporomandibular joint disorders. Dentomaxillofac Radiol. 2017 Jul;46(5):20160424

19. Shellock FG, Crues JV. MR procedures: biologic effects, safety, and patient care. Radiology. 2004 Sep;232(3):63552. Review.

20. Manfredini D, Bucci MB, Nardini LG. The diagnostic process for temporomandibular disorders. Stomatologija. 2007;9(2):35-9. Review.

21. Tomas X, Pomes J, Berenguer J, Quinto L, Nicolau C, Mercader JM, Castro V. MR imaging of temporomandibular joint dysfunction: a pictorial review.Radiographics. 2006 May-Jun;26(3):765-81. Review.

22. Milano V, Desiate A, Bellino R, Garofalo T. Magnetic resonance imaging of temporomandibular disorders: classification, prevalence and interpretation of disc displacement and deformation. Dentomaxillofac Radiol. 2000 Nov;29(6):352-61.

23. Rabelo KA, Sousa Melo SL, Torres MGG, Peixoto LR, Campos PSF, Rebello IMCR, de Melo DP. Assessment of condyle position, fossa morphology, and disk displacement in symptomatic patients. Oral Surg Oral Med Oral Pathol Oral Radiol. 2017 Aug;124(2):199-207.

24. Stanković S, Vlajković S, Bošković M, Radenković G, Antić V, Jevremović D.Morphological and biomechanical features of the temporomandibular joint disc: an overview of recent findings. Arch Oral Biol. 2013 Oct;58(10):1475-82. 